\title{
Statistical entropy of Schwarzschild black strings and black holes
}

\author{
Saurya Das* and Amit Ghosh ${ }^{\dagger}$ \\ Center for Gravitational Physics and Geometry, Department of Physics, The Pennsylvania State University, University Park, \\ Pennsylvania 16802-6300 \\ P. Mitra \\ Saha Institute of Nuclear Physics, 1/AF Bidhannagar, Calcutta 700064, India
}

(Received 1 June 2000; published 29 December 2000)

\begin{abstract}
The statistical entropy of a Schwarzschild black string in five dimensions is obtained by counting the black string states which form a representation of the near-horizon conformal symmetry with a central charge. The statistical entropy of the string agrees with its Bekenstein-Hawking entropy as well as that of the Schwarzschild black hole in four dimensions. The string length which gives the Virasoro algebra also reproduces the precise value of the Bekenstein-Hawking entropy and lies inside the stability bound of the string.
\end{abstract}

DOI: 10.1103/PhysRevD.63.024023

PACS number(s): 04.70.Dy, 04.50.+h, 11.25.Hf

That black hole solutions of general relativity have an entropy associated with them has been known for a long time, but statistical calculations have begun to appear relatively recently. So far statistical computations of entropy have mainly been limited to near-extremal black holes in a string theoretic approach [1] and non-rotating black holes in a quantum geometry approach [2].

The aim of this work was to encompass both nonextremal and extremal, both rotating and non-rotating black holes. There has been an attempt at finding a universally valid approach [3], and we have been inspired by it. A somewhat similar procedure has been given in [4]. Instead of using the detailed dynamics of the underlying quantum theory, these calculations rely more on symmetries. Our analysis follows the same general principle. However, in the detailed framework of [3], an essential restriction is made to the $(t-r)$-plane of black hole, i.e., the angular directions are disregarded. To us this seems artificial. Moreover, [3] is not directly applicable to nonrotating black holes. Here we set up a somewhat different way of getting a similar infinite conformal symmetry which can be directly used for a nonrotating black hole and can be generalized to the rotating case. This approach, like that of the earlier work, is also generalizable to arbitrary dimensions.

To count all microstates of a Schwarzschild black hole, we shall start with a five dimensional Schwarzschild black string. The Bekenstein-Hawking (BH) entropy of the string is the same as that of a Schwarzschild black hole in a spacetime of one lower dimension (because $G_{n}=l G_{n-1}$ ). We show that the Boltzmann entropy of the string agrees with its BH entropy and argue how one may reproduce the Boltzmann entropy of the black hole from that of the string.

We will consider a class of spacetimes whose nearhorizon geometry resembles that of a Schwarzschild string. We then consider spacetime diffeomorphisms which preserve the boundary conditions at the horizon. The key as-

\footnotetext{
*Email address: das@gravity.phys.psu.edu

†Email address: ghosh@gravity.phys.psu.edu

‡Email address: mitra@tnp.saha.ernet.in
}

sumption is that the near-horizon symmetries which give rise to non-zero "charges" will be realized as symmetries also in the quantum theory. The quantum numbers of these charges would then label the quantum states corresponding to the classical spacetime. It is in this spirit that one may count the degeneracy of a subset of states that are associated with a black hole of a given mass. A similar criterion was advocated in [5] for asymptotic symmetries. The canonical algebra of charges can be realized (in the physical phase space of general relativity) as a Virasoro algebra with a central extension. The entropy of the string is then given by the logarithm of the degeneracy of the representative states of the Virasoro algebra that share a common (large) conformal weight. A large conformal weight is equivalent to a large mass or area of the black string.

The line element of a Schwarzschild-string in "tortoise" coordinates is

$$
\begin{aligned}
d s^{2} & =\Delta\left(-d t^{2}+d r_{*}^{2}\right)+r^{2} d \theta^{2}+r^{2} \sin ^{2} \theta d \phi^{2}+l^{2} d \chi^{2}, \\
\Delta & =\frac{d r}{d r_{*}}=1-\frac{2 G M}{r} .
\end{aligned}
$$

Here $0 \leqslant \chi<1$ and thus $l$ is the length of the string. $M$ is the mass per unit length of the string. For the moment we keep $l$ arbitrary. We will consider the class of metrics which approach the Schwarzschild-string geometry near the horizon of Eq. (1). Our boundary conditions are motivated by the requirement of conformal symmetry:

$$
\begin{aligned}
\delta g_{t t} & =\mathcal{O}\left(\Delta^{2}\right), \quad \delta g_{t r_{*}}=\mathcal{O}(\Delta), \quad \delta g_{t \theta}=\mathcal{O}\left(\Delta^{2}\right), \\
\delta g_{t \phi} & =\mathcal{O}(\Delta), \quad \delta g_{t \chi}=\mathcal{O}(\Delta), \\
\delta g_{r_{*} r_{*}} & =\mathcal{O}(\Delta), \quad \delta g_{r_{*} \theta}=\mathcal{O}(\Delta), \quad \delta g_{r_{*} \phi}=\mathcal{O}(\Delta), \\
\delta g_{r_{*} \chi} & =\mathcal{O}(\Delta), \quad \delta g_{\theta \theta}=\mathcal{O}\left(\Delta^{2}\right), \\
\delta g_{\theta \phi} & =\mathcal{O}(\Delta), \quad \delta g_{\theta \chi}=\mathcal{O}(\Delta), \quad \delta g_{\phi \phi}=\mathcal{O}(\Delta), \\
\delta g_{\phi \chi} & =\mathcal{O}(\Delta), \quad \delta g_{\chi \chi}=\mathcal{O}(\Delta) .
\end{aligned}
$$


Now we seek vector fields $\xi^{\mu}$ which generate diffeomorphisms preserving these fall-off conditions. Let us make a near-horizon expansion of such a $\xi^{\mu}\left(t, r_{*}, \theta, \phi, \chi\right)$, which is a candidate for a near-horizon symmetry vector, in powers of $\Delta$ :

$$
\begin{aligned}
& \xi^{t}=T(t, \theta, \phi, \chi)+\mathcal{O}(\Delta), \quad \xi^{r_{*}=R(t, \theta, \phi, \chi)}+\mathcal{O}(\Delta), \\
& \xi^{\chi}=X(t, \theta, \phi, \chi)+\mathcal{O}(\Delta), \\
& \xi^{\theta}=\Theta(t, \theta, \phi, \chi)+\Theta_{1}(t, \theta, \phi, \chi) \Delta+\mathcal{O}\left(\Delta^{2}\right), \\
& \xi^{\phi}=\Phi(t, \theta, \phi, \chi)+\mathcal{O}(\Delta) .
\end{aligned}
$$

Expansion coefficients which are associated with nonvanishing on-shell ${ }^{1}$ surface charges (see below) at the horizon are physical and measurable, and those which give zero surface charges are irrelevant. The expansions (3) are motivated by the desire to get an infinite symmetry with the above requirement.

Equating $\delta g_{\mu \nu}=\mathcal{L}_{\xi} g_{\mu \nu}$ we get relations between various components of $\xi^{\mu}$,

$$
\begin{aligned}
t t: R & =-4 G M \partial_{t} T ; \\
t \theta: \partial_{t} \Theta & =0, \partial_{\theta} T=4 G M^{2} \partial_{t} \Theta_{1} ; \quad t \phi: \partial_{t} \Phi=0 ; \\
t \chi: \partial_{t} X & =0 ; \quad \theta \theta: \partial_{\theta} \Theta=0, R=-2 G M \partial_{\theta} \Theta_{1} ; \\
\theta \phi: \partial_{\phi} \Theta+\sin ^{2} \theta \partial_{\theta} \Phi & =0 ; \\
\theta \chi: \partial_{\theta} X & =\partial_{\chi} \Theta=0 ; \quad \phi \phi: \partial_{\phi} \Phi+\cot \theta \Theta=0 ; \\
\phi \chi: \partial_{\phi} X & =\partial_{\chi} \Phi=0 ; \\
\chi \chi: \partial_{\chi} X & =0,
\end{aligned}
$$

which can be solved completely. Solutions for $\Theta, \Phi, X$ and the zero mode of $\xi^{t}$ are

$$
\begin{aligned}
& \xi^{t}=T_{0}, \quad \Theta=A \cos \phi+B \sin \phi, \\
& \Phi=\cot \theta(-A \sin \phi+B \cos \phi)+k_{1}, \quad X=k_{2} .
\end{aligned}
$$

$T_{0}, A, B, k_{1}, k_{2}$ are constants. The following linear combinations of these form the five global Killing vectors of the Schwarzschild string (one associated with its mass per unit length, three associated with the rotational symmetry and one more with the translational symmetry along the length of the string): $\partial / \partial t, \partial / \partial \phi,(\cos \phi \partial / \partial \theta-\cot \theta \sin \phi \partial / \partial \phi),(\sin \phi \partial / \partial \theta$ $+\cot \theta \cos \phi \partial / \partial \phi), \partial / \partial \chi$,. For us, however, the interesting solutions are the higher order modes which are near-horizon symmetry vectors. It is convenient to express these near-

\footnotetext{
1، On-shell', refers to the implementation of the energy and momentum constraints. This phrase is to be distinguished from "onthe-solution," i.e., "on (1)," which will also be used here. Eventually all charges will be evaluated on-the-solution.
}

horizon symmetries in the Fourier modes of $\theta, \phi$ and $\chi$. If we define $x_{ \pm}=t / \sqrt{8 G^{2} M^{2}} \pm \theta$, one set of modes is

$$
\begin{aligned}
& T^{n n^{\prime} n^{\prime \prime}}=\sqrt{2 G^{2} M^{2}} \exp \left[2 i n x_{-}+i n^{\prime} \phi+2 \pi i n^{\prime \prime} \chi\right], \\
& R^{n n^{\prime} n^{\prime \prime}}=-4 G M i n \exp \left[2 i n x_{-}+i n^{\prime} \phi+2 \pi i n^{\prime \prime} \chi\right], \\
& \Theta_{1}^{n n^{\prime} n^{\prime \prime}}=-\exp \left[2 i n x_{-}+i n^{\prime} \phi+2 \pi i n^{\prime \prime} \chi\right] .
\end{aligned}
$$

There is another set involving $x_{+}$, which is obtained by replacing $x_{-}$by $x_{+}$. Its contribution will be incorporated later. The overall normalization of the solutions (6) is fixed by the surface-deformation (SD) algebra defined below. The $\phi$-mode number $n^{\prime}$ plays no interesting rôle in the analysis to follow and is set equal to zero. On the other hand, we pick up the diagonal elements $\left(n=n^{\prime \prime}\right)$ of $x_{-}$and $\chi$-modes in which $\xi^{\mu}$ furnish a $\operatorname{Diff}\left(S^{1}\right)$ algebra. If we define the surface deformation parameters $\hat{\xi}_{n}^{t}=\sqrt{\Delta} \xi_{n 0 n}^{t}$ and $\hat{\xi}_{n}^{i}=\xi_{n 0 n}^{i}$, the SD brackets [5] of $\hat{\xi}_{n}^{\mu}$ give rise to the corresponding brackets for $\xi^{\mu}$ in the following way:

$$
\begin{aligned}
& \left\{\hat{\xi}_{n}, \hat{\xi}_{m}\right\}_{\mathrm{SD}}^{t}=\hat{\xi}_{n}^{i} \partial_{i} \hat{\xi}_{m}^{t}-(m \leftrightarrow n), \\
& \left\{\xi_{n}, \xi_{m}\right\}_{\mathrm{SD}}^{t}=i(m-n) \xi_{m+n}^{t}, \\
& \left\{\hat{\xi}_{n}, \hat{\xi}_{m}\right\}_{\mathrm{SD}}^{i}=\hat{\xi}_{n}^{j} \partial_{j} \hat{\xi}_{m}^{i}+h^{i j} \hat{\xi}_{n}^{t} \partial_{j} \hat{\xi}_{m}^{t}-(m \leftrightarrow n), \\
& \left\{\xi_{n}, \xi_{m}\right\}_{\mathrm{SD}}^{i}=\mathcal{O}(\Delta)
\end{aligned}
$$

where $\quad\left\{\hat{\xi}_{n}, \hat{\xi}_{m}\right\}_{\mathrm{SD}}^{t}=\sqrt{\Delta}\left\{\xi_{n}, \xi_{m}\right\}_{\mathrm{SD}}^{t} \quad$ and $\quad\left\{\hat{\xi}_{n}, \hat{\xi}_{m}\right\}_{\mathrm{SD}}^{i}$ $=\left\{\xi_{n}, \xi_{m}\right\}_{\mathrm{SD}}^{i}$.

To realize these local symmetries in terms of the canonical Poisson brackets, let us recall the canonical surface deformation generators of the ADM formulation [6] [the phase space coordinates are $\left.\left(h_{i j}, \pi^{i j}\right)\right]$ :

$$
H[\hat{\xi}]=\frac{1}{16 \pi l G} \int d^{4} x \hat{\xi}^{\mu}(x) \mathcal{C}_{\mu}(x)+Q[\hat{\xi}]
$$

Here $\mathcal{C}_{\mu}=\left(\mathcal{C}, C_{i}\right)$ are the energy and momentum constraints $\mathcal{C}=\left(\pi_{i j} \pi^{i j}-\frac{1}{3} \pi^{2}\right) / \sqrt{h}-{ }^{4} R \sqrt{h}$ and $C_{i}=-2 \pi_{i \mid j}^{j}$, where ${ }^{4} R$ is the curvature of the $t=$ constant surface and $\pi=\pi_{i}^{i}$. A vertical bar denotes covariant differentiation with respect to the induced metric on the space slice. $Q[\hat{\xi}]$ represents the appropriate boundary term which, in the presence of the boundary conditions (2), makes the total generator $H[\hat{\xi}]$ differentiable at the boundary. In other words, boundary terms appearing due to the variation of the constraints in $H[\hat{\xi}]$ in the phase space coordinates and from variations of $Q[\hat{\xi}]$ cancel each other in the presence of the boundary conditions (2). The variation of the constraints alone thus gives the variation of $\delta Q[\hat{\xi}]$ as a combination of total derivatives:

$$
\begin{aligned}
\delta Q[\hat{\xi}]= & \frac{1}{16 \pi l G} \int d^{4} x\left\{G^{i j k l}\left[\hat{\xi}^{t}\left(\delta h_{i j}\right)_{\mid k}-\delta h_{i j} \hat{\xi}_{, k}^{t}\right]\right. \\
& \left.+2 \hat{\xi}^{i} \delta \pi_{i}^{l}-\hat{\xi}^{l} \pi^{i k} \delta h_{i k}\right\}_{\mid l}
\end{aligned}
$$


where $2 G^{i j k l}=\sqrt{h}\left(h^{i k} h^{j l}+h^{i l} h^{j k}-2 h^{i j} h^{k l}\right)$ and the integral is evaluated at the horizon of Eq. (1). The variations $\left(\delta h_{i j}, \delta \pi^{i j}\right)$ in Eq. (9) belong to the constraint surface in the phase space while the coefficients take their values on the solution. The coefficient of the last term vanishes identically on-the-solution. The rest of the variations can be integrated, giving rise to the surface charge

$$
Q[\hat{\xi}]=\frac{1}{16 \pi l G} \int d^{4} x\left\{\bar{G}^{i j k l}\left[\hat{\xi}^{t} h_{i j \mid k}-h_{i j} \hat{\xi}_{, k}^{t}\right]+2 \hat{\xi}^{i} \pi_{i}^{l}\right\}_{\mid l}
$$

where all barred quantities refer to the on-the-solution metric. The surface charge (10) simplifies on-the-solution to

$$
Q[\hat{\xi}]=\frac{1}{16 \pi l G} \int_{\Delta} d \theta d \phi d \chi\left\{\bar{G}^{i j k r_{*}}\left[-\bar{h}_{i j} \partial_{k} \hat{\xi}^{t}\right]\right\} .
$$

Since the surface charges (11) are linear functionals of $\xi^{\mu}$, they obey the canonical Poisson brackets algebra induced by the SD algebra (7) in the constrained phase space $\{Q[\hat{\xi}], Q[\hat{\eta}]\}=Q\left[\{\hat{\xi}, \hat{\eta}\}_{\mathrm{SD}}\right]+W_{\xi \eta}$ where $W$ is a possible central extension. The canonical Poisson bracket can be cast into the form of a Lie bracket

$$
\left\{Q\left[\hat{\xi}_{n}\right], Q\left[\hat{\xi}_{m}\right]\right\}=Q\left[\left\{\hat{\xi}_{n}, \hat{\xi}_{m}\right\}_{\mathrm{SD}}\right]+W_{n m}=\mathcal{L}_{\hat{\xi}_{m}} Q\left[\hat{\xi}_{n}\right],
$$

where $\mathcal{L}_{\hat{\xi}_{n}} \hat{\xi}_{m}^{t}=\hat{\xi}_{n}^{i} \partial_{i} \hat{\xi}_{m}^{t}$. Equation (12) can be understood as follows. In phase space one can associate a vector field $q^{a}\left[h_{i j}, \pi^{i j}\right]$ with each phase space scalar functional, say the charge $Q$, such that $\mathcal{L}_{q} F=\{Q, F\}$, for an arbitrary scalar functional $F$. Since $Q$ is a linear functional of the phase space coordinates [see Eq. (10)] and $\hat{\xi}^{\mu}$, the Lie derivative generated by the vector field $q^{a}$ is taken to be equal to $\mathcal{L}_{\hat{\xi}}$.

The (infinitely many) canonical generators are obtained by using the various modes of $\hat{\xi}^{\mu}$ in Eq. (11):

$$
\begin{aligned}
L_{n} & =Q\left[\hat{\xi}_{n}\right] \\
& =\frac{1}{8 \pi l G} \int_{\Delta} d \theta d \phi d \chi\left(\sqrt{h} h^{r_{*}{ }^{*} \partial_{r_{*}}} \hat{\xi}_{n}^{t}\right) \\
& =\frac{A_{H} \sqrt{2}}{32 \pi G} \delta_{n 0},
\end{aligned}
$$

where $A_{H}=16 \pi(G M)^{2}$. The central extension $W_{n m}$ is evaluated from the formula (12) and the SD bracket (7):

$$
W_{n m}=i(n-m) L_{m+n}+\mathcal{L}_{\hat{\xi}_{m}} Q\left[\hat{\xi}_{n}\right] .
$$

In quantum theory we replace the Poisson brackets by commutator brackets: $i\{\ldots\} \rightarrow[\ldots]$. As a result the central charge takes the following form:

$$
i W_{n m}=i \mathcal{L}_{\hat{\xi}_{m}} Q\left[\hat{\xi}_{n}\right]-(n-m) L_{n+m}{ }^{\operatorname{def}} c \frac{c}{12}\left(n^{3}-n\right) \delta_{m+n} .
$$

Thus the value of $c$ depends on the on-the-solution value of $L_{n+m}$ and the Lie bracket

$$
\mathcal{L}_{\hat{\xi}_{m}} Q\left[\hat{\xi}_{n}\right]=\frac{1}{8 \pi l G} \int d r_{*} d \theta d \phi d \chi \sqrt{h} h^{k l} \hat{\xi}_{m}^{i} \nabla_{i} \nabla_{l} \partial_{k} \hat{\xi}_{n}^{t} .
$$

One comment is in order regarding the formula (16). The $r_{*}$ integral is evaluated at $r=2 G M$ (one also uses $d r=\Delta d r_{*}$ ). The required anti-symmetry of $W_{n m}$ under the exchange of $(m \leftrightarrow n)$ results naturally from the on-the-solution value of $L_{n+m}$ in Eq. (13) and the integral (16) which turns out to have the general form $\left(\gamma n^{3}+\beta n\right) \delta_{m+n}$, where $\gamma=\left(\alpha^{2}\right.$ -1) $A_{H} \sqrt{2} / 4 \pi G$ and $\beta=A_{H} \sqrt{2} / 32 \pi G$ with $\alpha=2 \pi G M / l$. Note that a linear term in $n$ appears also from $L_{n+m}$. Upon adding the two linear terms, one requires the $\left(n^{3}-n\right)$ form of the central extension which is needed to make $W_{1,-1}=0$. This can be achieved by shifting $L_{0}$, or more simply by the choice $\gamma=\beta$, i.e., $\alpha^{2}=9 / 8$. The value of $c$ is then read off from the coefficient $\gamma$ :

$$
c=12 \gamma=\frac{3 A_{H} \sqrt{2}}{8 \pi G} .
$$

It is necessary to check the stability of the string [7] (see also [8] for an entropy argument). The choice $\alpha^{2}=9 / 8$ leads to $3 l=4 \pi G M \sqrt{2}$ which is inside the stability bound $0<l$ $<3.375 \pi G M$ (estimated from the entropy bound $S_{\text {string }}>S$ (5D Schwarzschild of mass $M$ ).

On using Eqs. (17) and (13) the statistical entropy $S_{-}$ from the $\left(x_{-}\right)$-sector is found to be given (for $A_{H} \gg G$ ) [9] by

$$
S_{-}=2 \pi \sqrt{\frac{c L_{0}}{6}}=\frac{A_{H}}{8 G} .
$$

The two sectors, $x_{ \pm}$, thus give the total entropy $S=S_{-}$ $+S_{+}=A_{H} / 4 G$ which agrees with the Bekenstein-Hawking entropy.

We end with a few comments on some aspects of the calculation. (1) We chose a specific set of boundary conditions near the horizon. The choice was motivated by $[1,3,10]$ where it was shown that conformal symmetry plays a key rôle in describing near-horizon states. Our primary aim has been to obtain the conformal symmetry. (2) Our boundary condition (2) is only one of possible choices, but it is one that gives a conformal symmetry with non-vanishing central charge. Presumably it provides the maximum degeneracy. (3) The counting of black hole states is reproduced by the counting of black string states in the following sense: the Boltzmann entropies of the black string and the black hole should be related as $\log \Omega_{\text {string }} \sim \log \Omega_{\text {hole }}+N$ where $N$ is the number of microscopic constituents along the string which is proportional to its length $l$ which in turn is proportional to $M$. Thus the statistical entropy of the black hole is $S$ $\sim \log \Omega_{\text {string }}-l \sim M^{2}-M \sim M^{2}$ for large $M$, which matches the Bekenstein-Hawking entropy of the black hole. (4) We require a large string length in the above calculation, implying a five dimensional Planck length $\left(l_{P} l\right)^{1 / 2}$ which is large 
compared to the four dimensional Planck length $l_{P}$. This signals the opening up of an extra dimension near the horizon [11], though what happens asymptotically is not clear at the moment. (5) The conformal symmetry lives in the plane $\left(t / \sqrt{8 G^{2} M^{2}}+2 \pi \chi\right) \pm \theta$. One may be tempted to find a connection between this plane and the string world sheet. However, the $\left(t-r_{*}\right)$-plane plays no special rôle in this approach and both the sectors of conformal symmetry contribute equally to the entropy, unlike in [3]. (6) The leading order correction to the entropy is logarithmic (coming from the power-law corections to Cardy formula): $S \sim \log \left(A_{H} / 4 G\right)$, as discussed by [12] in detail.

S.D. and A.G. thank Abhay Ashtekar for discussions and various suggestions. They also thank P. Majumdar for discussions on corrections to the area formula. The work of S.D. and A.G. was supported by the National Science Foundation grant PHY95-14240 and the Eberly Research Funds of Penn State. P.M. thanks the Theory Division of CERN, where the collaboration started.
[1] A. Strominger and C. Vafa, Phys. Lett. B 379, 99 (1996); J. Maldacena, Ph.D. thesis, Princeton University, hep-th/9607235.

[2] A. Ashtekar, J. Baez, A. Corichi, and K. Krasnov, Phys. Rev. Lett. 80, 904 (1998); A. Ashtekar, J. Baez, and K. Krasnov, Report No. NSF-ITP-99-153.

[3] S. Carlip, Phys. Rev. Lett. 82, 2828 (1999); Class. Quantum Grav. 16, 3327 (1999).

[4] S. Solodukhin, Phys. Lett. B 454, 213 (1999).

[5] C. Teitelboim, Ann. Phys. (N.Y.) 79, 542 (1973); J. Brown and M. Henneaux, Commun. Math. Phys. 104, 207 (1986); M. Henneaux and C. Teitelboim, ibid. 98, 391 (1985).

[6] T. Regge and C. Teitelboim, Ann. Phys. (N.Y.) 88, 286
(1974).

[7] R. Gregory and R. Laflamme, Phys. Rev. Lett. 70, 2837 (1993); Nucl. Phys. B428, 399 (1994).

[8] G. Horowitz and E. Martinec, Phys. Rev. D 57, 4935 (1998).

[9] J. L. Cardy, Nucl. Phys. B270, 186 (1986).

[10] G. 't Hooft, Acta Phys. Pol. B 19, 187 (1988); Phys. Scr. T15, 143 (1987); Nucl. Phys. B256, 727 (1985); A. Strominger, J. High Energy Phys. 02, 009 (1998).

[11] I. Antoniadis, N. Arkani-Hamed, S. Dimopoulos, and G. Dvali, Phys. Lett. B 436, 257 (1998); L. Randall and R. Sundrum, Phys. Rev. Lett. 83, 4690 (1999).

[12] S. Carlip, Class. Quantum Grav. 17, 4175 (2000); R. K. Kaul and P. Majumdar, Phys. Rev. Lett. 84, 5255 (2000). 